\title{
Coupled flood and sediment transport modelling with adaptive mesh refinement
}

\author{
HUANG Wei ${ }^{1}$, CAO ZhiXian ${ }^{1,2^{*}}$, PENDER Gareth $^{1,2}$, LIU QingQuan $^{3}$ \& CARLING Paul ${ }^{4}$ \\ ${ }^{1}$ State Key Laboratory of Water Resources and Hydropower Engineering Science, Wuhan University, Wuhan 430072, China; \\ ${ }^{2}$ Institute for Infrastructure and Environment, Heriot-Watt University, Edinburgh EH14 4AS, UK; \\ ${ }^{3}$ Institute of Mechanics, Chinese Academy of Sciences, Beijing 100190, China; \\ ${ }^{4}$ Geography \& Environment, University of Southampton, Southampton SO17 1BJ, UK
}

Received December 21, 2014; accepted May 10, 2015; published online July 8, 2015

\begin{abstract}
Coupled flood and sediment transport modelling in large-scale domains has for long been hindered by the high computational cost. Adaptive mesh refinement is one of the viable ways to solving this problem without degrading the accuracy. This goal can be accomplished through mesh adaptation, e.g., mesh coarsening and refining based on the dynamic regime of the flow and sediment transport along with bed evolution. However, previous studies in this regard have been limited to cases either without involving sediment transport or featuring flow-sediment-bed decoupling and the assumption of sediment transport capacity, which are not generally justified. Here, a coupled hydrodynamic and non-capacity sediment transport model is developed on adaptive non-uniform rectangular mesh. The proposed model is validated against experimental tests and numerical results based on the fixed meshes. It is demonstrated that the proposed model can properly capture shock waves, resolve the wetting/drying transition and reproduce morphological evolution. Compared with models based on the fixed meshes, the proposed model features great advantage in computational efficiency and holds promise for wide applications.
\end{abstract}

shallow water flow, sediment transport, adaptive mesh

Citation: Huang W, Cao Z X, Pender G, et al. Coupled flood and sediment transport modelling with adaptive mesh refinement. Sci China Tech Sci, 2015, 58: 1425-1438, doi: 10.1007/s11431-015-5880-6

\section{Introduction}

To underpin effective flood risk management, it is necessary to numerically model flood flows that involve active sediment transport and morphological evolution [1,2]. Despite the rapid development of computer technology and numerical algorithms in the last several decades, the high computational cost continues to hinder the wide applications of full two-dimensional (2D) shallow water hydrodynamic modelling in large-scale domains [3,4].

Generally, a high-resolution mesh is required in order to

\footnotetext{
*Corresponding author (email: zxcao@whu.edu.cn)
}

ensure the accuracy and reliability of the modelling. For example, buildings are in general densely distributed in an urban area and thus a high-resolution mesh is normally required in urban inundation modelling [5-7]. Local flow regime is important for fish habitat in ecological studies, and in this connection Crowder and Diplas [8] employed a locally fine mesh to resolve large boulders in natural rivers. However, a high-resolution mesh means a large number of computational nodes and thus a huge increase in computational cost [9]. In general, for large-scale hydrodynamic modelling, the need for a high-resolution mesh exists only in a small portion of the entire domain, while over the majority of the domain a locally coarse mesh suffices. This implies that part of the computational nodes can be removed 
without degrading the overall accuracy. Therefore, mesh optimization, i.e., applying a high-resolution mesh only in those areas of major interest, may significantly improve the computational efficiency while at the same time maintain the reliability of the solution. On the other hand, the flow usually evolves dynamically in space and time. Therefore, the area in which a locally high-resolution mesh should be adopted is unknown, which necessitates the use of adaptive mesh refinement (AMR). The most widely used structured AMR was developed by Berger and Oliger [9]. Based on the AMR algorithm, they developed a model of $2 \mathrm{D}$ hyperbolic partial differential equations. Following Berger and Oliger [9], George [10] employed block-structured AMR to develop a 2D shallow water hydrodynamic model for tsunami modelling. The ratio of the mesh sizes between distinct levels was arbitrary, which differs from that of the traditional quadtree mesh $[10,11]$ that restricted the ratio between levels to 2.0. Chen et al. [12] developed a non-inertia model based on a multi-layered mesh for urban inundation modelling. However, it is a lumped model, which neglects the dynamics of the flood to a certain extent. Liang [4] developed a structured non-uniform AMR for 2D shallow water hydrodynamic modelling over fixed bed. Compared with the block-structured AMR and traditional quadtree mesh [9-11,13], this mesh features advantages in neighbour search, cell information storage and adaptation procedure.

It is recognized that environmental flows may induce active sediment transport and morphological evolution, which in turn modify the flows. One of the most telling cases is dam-break flow over an erodible sediment bed [14-16]. To date, however, most studies using AMR have been limited to cases either without involving sediment transport at all $[4,9-11,13,17]$ or featuring the assumption of sediment transport capacity, which is not normally justified. Benkhaldoun et al. [18] have developed a model based on an unstructured AMR for modelling of flood flows over mobile beds. Compared with the structured AMR, the generation of the unstructured AMR is more complex and the discretization of the governing equations becomes much more cumbersome. Further, the model is decoupled, neglecting the feedback impacts of sediment transport and morphological evolution on the flow to a certain extent. Equally critically, it features the assumption of sediment transport capacity, i.e., sediment transport rate is assumed to be determined exclusively by local flow regime. However, this assumption is not generally justified, especially in highly unsteady flows such as dam-break flows over mobile beds [15]. Zhang and $\mathrm{Wu}$ [19] developed a hydrodynamic and sediment transport model based on a multiple-level quadtree mesh with local refinement for the dam-break floods over mobile bed. However, the mesh was fixed during the modelling and the location with local mesh refinement was predefined. The authors were not aware of any coupled hydrodynamic and non-capacity sediment transport model featuring adaptive mesh refinement.

In the present paper, a coupled hydrodynamic and non-capacity sediment transport model on an adaptive non-uniform rectangular mesh is proposed. It is an extension of the model by Huang et al. [20] on a fixed uniform mesh to the adaptive non-uniform rectangular mesh similar to Liang [4]. A new adaptive indicator is introduced to reflect the impact of sediment concentration on the AMR. The Godunov-type finite volume method with Harten-Lax-van Leer contact (HLLC) approximate Riemann solver and monotonic upstream-centered scheme for conservation laws (MUSCL) linear reconstruction are deployed to discretize the governing equations. Several laboratory experimental cases are modelled to validate the proposed model, including dam break floods over both the fixed and mobile beds. The proposed model is finally applied to model a megaflood due to glacier dam break in a large-scale domain. The accuracy and computational efficiency of the present model are demonstrated.

\section{Mathematical model}

\subsection{Governing equations}

The governing equations are essentially the $2 \mathrm{D}$ shallow water hydrodynamic equations comprising the complete mass and momentum conservation equations for the watersediment mixture flow and the mass conservation equations for sediment and bed material, respectively. The wellbalanced conservative form of the governing equations is written as [20]

$$
\begin{gathered}
\frac{\partial \boldsymbol{U}}{\partial t}+\frac{\partial \boldsymbol{F}}{\partial x}+\frac{\partial \boldsymbol{G}}{\partial y}=\boldsymbol{S}, \\
\boldsymbol{U}=\left[\begin{array}{l}
\eta \\
h u \\
h v \\
h c
\end{array}\right], \\
\boldsymbol{F}=\left[\begin{array}{c}
h u \\
h u^{2}+0.5 g\left(\eta^{2}-2 \eta z\right) \\
h u v \\
h u c \\
h v \\
h v \\
h u v \\
h v^{2}+0.5 g\left(\eta^{2}-2 \eta z\right) \\
h v c
\end{array}\right],
\end{gathered}
$$




$$
\begin{gathered}
\boldsymbol{S}=\boldsymbol{S}_{s}+\boldsymbol{S}_{f}=\left[\begin{array}{c}
0 \\
-g \eta \frac{\partial z}{\partial x} \\
-g \eta \frac{\partial z}{\partial y} \\
0
\end{array}\right] \\
+\left[\begin{array}{c}
\rho \\
-\frac{\tau_{b x}}{\rho}+\varepsilon \nabla^{2}(h u)-\frac{\Delta \rho}{2 \rho} \frac{g h^{2} \partial c}{\partial x}-\frac{\left(\rho_{0}-\rho\right)(E-D) u}{\rho\left(1-p_{0}\right)} \\
-\frac{\Delta \rho}{\rho}+\varepsilon \nabla^{2}(h v)-\frac{\Delta \rho}{2 \rho} \frac{g h^{2} \partial c}{\partial y}-\frac{\left(\rho_{0}-\rho\right)(E-D) v}{\rho\left(1-p_{0}\right)} \\
\frac{\partial z}{\partial t}=\frac{D-E}{1-p_{0}}
\end{array}\right]
\end{gathered}
$$

where $\boldsymbol{U}$ is a vector of conserved variables; $\boldsymbol{F}$ and $\boldsymbol{G}$ are the convective flux vectors of the flow in $x$ - and $y$-directions, respectively; $\boldsymbol{S}$ is the source term including the bed slope $\boldsymbol{S}_{s}$, and the friction source term $\boldsymbol{S}_{f}$ that incorporates the friction stresses and other terms related to sediment transport and bed evolution; $\eta$ is the water surface level above the datum; $z$ is the bed elevation; $h$ is the water depth; $u$ and $v$ are velocity components in $x$ - and $y$-directions, respectively; $c$ is the depth averaged volumetric sediment concentration; $g$ is the gravitational acceleration; $p_{0}$ is the bed sediment porosity; $\Delta \rho=\rho_{s}-\rho_{w}$, where $\rho_{w}$ and $\rho_{s}$ are densities of water and sediment, respectively; $\rho$ and $\rho_{0}$ are the densities of water-sediment mixture and saturated bed material, where $\rho=\rho_{w}(1-c)+\rho_{s} c$ and $\rho_{0}=\rho_{w} p_{0}+\rho_{s}\left(1-p_{0}\right)$; $E$ and $D$ are sediment entrainment and deposition fluxes across the bottom boundary of flow, representing the sediment exchange between the water column and river bed; $\tau_{b x}$ and $\tau_{b y}$ are bed friction stresses; $\nabla^{2}$ is the Laplace operator; $\varepsilon$ is the turbulent eddy viscosity, which is calculated by $\varepsilon=\gamma \kappa h u_{*}$, where $\gamma$ is a coefficient, $\kappa$ is the von Karman coefficient, and $u_{*}$ is friction velocity.

\subsection{Model closure}

To close the governing equations, a set of empirical relationships is introduced to determine the bed shear stress and the sediment exchange fluxes. In general, environmental flows are unsteady and non-uniform, featuring boundary resistance substantially different from those of steady and uniform flows. This fact is more pronounced where sediment transport is involved and the bed is rendered mobile and exhibits bedforms (e.g., dunes). Unfortunately, there has been no generally applicable relationship for boundary resistance in such flows, which has been proved to result in improved results. This is why computational studies of environmental flows over fixed and mobile beds have to date exclusively used resistance relationships initially developed for steady and uniform flows, which involves the Manning roughness $n$. The present model follows the same practice in this aspect, the bed shear stresses are given by

$$
\begin{aligned}
& \tau_{b x}=\rho g n^{2} u \sqrt{u^{2}+v^{2}} / h^{1 / 3}, \\
& \tau_{b y}=\rho g n^{2} v \sqrt{u^{2}+v^{2}} / h^{1 / 3} .
\end{aligned}
$$

Two distinct mechanisms are involved in the sediment exchange between the flow and bed, i.e., sediment entrainment due to turbulence and particle-particle interactions, and sediment deposition mainly due to gravitational action. The entrainment and deposition fluxes are estimated by

$$
\begin{gathered}
E=\alpha \omega c_{e}, \\
D=\alpha \omega c,
\end{gathered}
$$

where $\omega$ is the settling velocity of a single sediment particle in tranquil clear water, which is calculated using the formula of Zhang and Xie [21]. Bed load sediment transport capacity $c_{e}$ is determined by local flow regime. The empirical parameter $\alpha$ represents the difference between the near-bed concentration and the depth-averaged concentration of sediment; its value is determined by the method in $\mathrm{Cao}$ et al. [15]. The bed load sediment transport capacity $c_{e}$ is given by

$$
\begin{gathered}
c_{e}=q_{b} / h \sqrt{u^{2}+v^{2}}, \\
q_{b}=\varphi 8 \sqrt{s g d^{3}}\left(\theta-\theta_{c}\right)^{1.5},
\end{gathered}
$$

where $q_{b}$ is unit-width bed load transport rate at transport capacity status; $s$ is the specific gravity of sediment; $d$ is the sediment medium diameter; $\theta$ is the Shields parameter and $\theta_{c}$ is threshold Shields parameter for initiation of sediment movement, determined following Cao et al. [22]. A plethora of empirical formulations has been proposed for $q_{b}$. Yet existing formulations for bed load transport rate are derived under steady and uniform flows, and therefore the entrainment flux based on these formulations may not be directly applicable to rapid flows (e.g., dam break floods). A slightly modified version of the MPM [23] formula is introduced, with a modification coefficient $\varphi$ to be calibrated using measured data, since the Shields parameter in dam break floods could go beyond the range in which the MPM formula was initially derived. In essence, it is a functional form of bed load transport rate introduced based on the MPM formula.

\subsection{Non-uniform rectangular mesh and adaptation}

The non-uniform rectangular mesh is implemented by using 
the AMR technique. It is implemented in three steps following Liang [4]. Firstly, the computational domain is decomposed using a coarse uniform rectangular mesh, referred to as the background mesh. Secondly, subdivision level is allocated to each cell on the background mesh according to specified criteria. Finally, the mesh is regularized to ensure that an arbitrary cell is at most two times bigger or smaller than any of its neighbors (i.e., the 2:1 rule).

After regularization of the mesh, the neighbours of each cell can be found by simple algebraic relationships following Liang [4], which is easier to implement than the traditional quadtree mesh [11,13]. Moreover, the present non-uniform mesh allows the modelling to be implemented in a single mesh unit without any need to divide the computation into different individual mesh components as required by a block-structured mesh. Also it does not necessitate troublesome dynamical links of different numerical solutions as for a nested mesh and quadtree mesh [4]. Generally, the initial mesh can be created by seeds and a looped boundary $[4,10]$. Alternatively, all the initial mesh of computational cases is allocated to the highest level for simplicity.

During the modelling, the mesh adaptation procedure can be implemented by refining and coarsening the mesh with a specified adaptation indicator. One issue common to all the AMR techniques is the selection of the adaptation indicator and its value. To date, there is no universally applicable approach to address this problem. Berger and Oliger [9] determined the adaptation indicator based on the difference of variables between two successive time instants. George [10] applied the flow criteria (such as water surface gradient and velocity) as adaptation indicator. In both Berger and Oliger [9] and George [10], if the adaptation indicator exceeds the pre-specified threshold, such as $\sqrt{u^{2}+v^{2}}>0.1$ $\mathrm{m} / \mathrm{s}$ in George [10], the cell would be flagged out for refinement. An alternative method for determining the adaptation indicator involves the relative value of the flow variables. Wang and Liang [17] used the nondimensionalized water surface gradient as an adaptation indicator. The threshold values for refinement and coarsening are obtained by sorting the array of the variables. In this regard, it is noted that the extra data storage or computation may be highly costly as the array is huge. Benkhaldoun et al. [18,24] employed the normalized pollution concentration or bed load transport rate as an adaptation indicator. In this method, if the normalized value exceeds the threshold, the cell is marked for refinement or coarsening. In the present work, the gradients of water surface and sediment concentration as well as the bed slope are employed to determine the adaptation indicator. The relative values are adopted as thresholds. The function for the adaptation indicator is defined as

$$
G(\psi) \equiv \sqrt{(\partial \psi / \partial x)^{2}+(\partial \psi / \partial y)^{2}},
$$

where $G$ is a functional symbol; $\psi$ represents either the wa- ter surface elevation $\eta$, bed elevation $z$ or the volumetric sediment concentration $c$. To define the thresholds of the adaptation indicator, the gradients are normalized as

$$
\sigma_{i c}=\frac{G_{i c}}{G_{\max }},
$$

where the subscript ic is the cell index; $G_{\max }$ is a maximum value among all the cells; $\sigma_{1}$ and $\sigma_{2}$ are two coefficients used to indicate the sensitivity of the mesh adaptation procedure. $\sigma_{1}$ is used for marking the cells that should be refined. For example, if $\sigma_{1}=0.8$, it means that the cells with $\sigma_{i c}>0.8$ should be subjected to mesh refinement. $\sigma_{2}$ is used for marking the cells to be coarsened. During the modelling, if the adaptation indicator for any of the three variables $(\eta, z, c)$ satisfies $\sigma_{i c}>\sigma_{1}$ and the level is smaller than the highest level, the cell is to be subdivided, i.e., lev $=\mathrm{lev}+1$. On the other hand, if all the subcells in cell $(i, j)$ meets $\sigma_{i c}<\sigma_{2}$ for all the three variables and the level is larger than zero, the cell is flagged to be coarsen (i.e., lev=lev-1). The values $\sigma_{1}$ and $\sigma_{2}$ are thresholds for refinement and coarsening and they are case dependent. A sensitive analysis has been conducted to evaluate to what extent the threshold values $\sigma_{1}$ and $\sigma_{2}$ affect the results. It is revealed (not shown here) that the two thresholds change the results marginally for the fixed beds, while discernibly for mobile beds. Generally, the two thresholds are larger for the fixed beds than those over mobile beds. In applications, the ranges of the $\sigma_{1} \in 0.5-0.8$ and $\sigma_{2} \in 0.1-0.3$ are suggested for cases over fixed beds, while $\sigma_{1} \in 0.2-0.4$ and $\sigma_{2} \in 0.01-0.1$ for cases over mobile beds. Additionally, the cells at the wet/dry interface are always subdivided to the highest levels but not confined by eq. (7). After its refinement and coarsening, the mesh must be regularized to satisfy the 2:1 rule. In any newly created cell, its flow and sediment transport variables are obtained by means of linear interpolation based on values at its neighbours following Liang [4].

\subsection{Numerical scheme}

An explicit finite volume Godunov-type scheme is adopted to solve the pre-balanced governing equations on the structured non-uniform rectangular mesh. Although the numerical scheme is designed for uniform fixed meshes, it is also applicable to the non-uniform mesh with moderate modifications. The HLLC approximated Riemann solver is used to solve the local Riemann problem and the interface fluxes and the MUSCL linear reconstruction is adopted to achieve the second-order accuracy in space.

The following time-marching formulae are used to update the flow and sediment variables to a new time step 
$(k+1)$ :

$$
\begin{gathered}
\boldsymbol{U}_{i c}^{p}=\boldsymbol{U}_{i c}^{k}+\Delta t \boldsymbol{S}_{f i c}^{k+1}, \\
\boldsymbol{U}_{i c}^{k+1}=\boldsymbol{U}_{i c}^{p}-\Delta t\left(\boldsymbol{F}_{E}-\boldsymbol{F}_{W}\right)^{p} / \Delta x_{s} \\
-\Delta t\left(\boldsymbol{G}_{N}-\boldsymbol{G}_{S}\right)^{p} / \Delta y_{s}+\Delta t \boldsymbol{S}_{s i c}^{k},
\end{gathered}
$$

where the superscript $k$ represents the time level and the superscript $p$ indicates the state after calculating variables from eq. (8); $\Delta t$ is the time step; $\Delta x_{s}$ and $\Delta y_{s}$ denote cell size in the $x$ - and $y$-directions; $\boldsymbol{F}_{E}, \boldsymbol{F}_{W}, \boldsymbol{G}_{N}$ and $\boldsymbol{G}_{S}$ are interface fluxes through the east, west, north and south directions. Taking $\boldsymbol{F}_{E}$ as an example, the interface fluxes may be calculated through solving the local Riemann problem defined at the eastern edge of cell ic,

$$
\boldsymbol{F}_{E}=\boldsymbol{F}\left(\boldsymbol{U}_{E}^{+}, \quad \boldsymbol{U}_{E}^{-}\right),
$$

where $\boldsymbol{U}_{E}^{+}$and $\boldsymbol{U}_{E}^{-}$are the reconstructed face values of the flow and sediment variables at right- and left hand -side of the cell edge under consideration. Based on the flow and sediment information stored at the cell centers, the MUSCL slope limited linear reconstruction is used to define the Riemann states, which is essentially the same as Huang et al. [20]:

$$
\boldsymbol{U}_{E}^{-}=\boldsymbol{U}_{i c}+0.5 \Delta x_{s} \nabla \boldsymbol{U}_{i c} \text { and } \boldsymbol{U}_{E}^{+}=\boldsymbol{U}_{e}-0.5 \Delta x_{s} \nabla \boldsymbol{U}_{e},
$$

where $\nabla \boldsymbol{U}_{i c}$ and $\nabla \boldsymbol{U}_{e}$ are the vectors of slope limited gradient evaluated at cell ic and its eastern neighbour. $\boldsymbol{U}_{e}$ contains the flow and sediment variables at the eastern neighbour cell. For simplicity, it is assumed that the eastern neighbour is of the same size with the cell under consideration. Otherwise, the calculation is implemented in the same way as that on a uniform mesh after interpolation [4]. $\nabla \boldsymbol{U}_{i c}$ is given by minmod slope limiter for better numerical stability [25]

$$
\begin{aligned}
& \nabla \boldsymbol{U}_{i c} \\
& =\min \bmod \left[\left(\boldsymbol{U}_{i c}-\boldsymbol{U}_{w}\right) / \Delta x_{s}, \quad\left(\boldsymbol{U}_{e}-\boldsymbol{U}_{i c}\right) / \Delta x_{s}\right],
\end{aligned}
$$

where $\boldsymbol{U}_{w}$ is the vector of the flow and sediment variables at the western neighbour. Riemann states at other cell interfaces are calculated similarly. It should be noted that the slope-limited reconstruction is only applicable to those wet cells away from the wet-dry interface. In a dry or a wet cell next to a dry cell $\left(h_{i c}<10^{-6} \mathrm{~m}\right)$, the face values are set to be equal to those at the centers to give a stable representation of the wet-dry fronts. The local Riemann problem defined by the above left and right Riemann states is solved by the HLLC approximate Riemann solver. The details about the HLLC Riemann solver can be referred to ref. [26].
Due to the different sizes of the neighbours, special care must be taken to maintain the conservative property as evaluating interface fluxes [4]. Figure 1 illustrates a typical case, where cell $P$ has two eastern neighbours with smaller size. The flux vector $\boldsymbol{F}_{E}$ through the eastern interface is not directly evaluated, but computed as $\boldsymbol{F}_{E}=\left(\boldsymbol{F}_{E 1}+\boldsymbol{F}_{E 2}\right)$, herein $\boldsymbol{F}_{E 1}$ and $\boldsymbol{F}_{E 2}$ are the fluxes through the mid-point of the western interface of the two smaller size neighbour cells. This treatment guarantees the mass and momentum conservation of the finite volume method. The flux vectors in other directions can be calculated similarly. The bed slope is discretized by central difference approach, take $x$-direction as example

$$
-g \eta \partial z / \partial x=-g \bar{\eta}\left(z_{E}-z_{W}\right) / \Delta x_{i c}
$$

where $\bar{\eta}=0.5\left(\eta_{E}^{L}+\eta_{W}^{R}\right)$. Similar discretization is applied to the $y$-direction. This treatment can balance the source term and the flux gradient, which preserves the well balanced property of the model at static water condition (Liang and Marche [27]). For the friction source term $S_{f}$, the splitting implicit method is used with limited friction force, which can avoid nonphysical velocity as the depth is very small [27]. In eq. (8), the friction source term $S_{f}^{k+1}$ is solved by a splitting method and it is equivalent to solving the following ordinary differential equations

$$
\frac{\mathrm{d} \boldsymbol{U}}{\mathrm{d} t}=\boldsymbol{S}_{f}
$$

Take the momentum equation of eq. (14) in the $x$-direction as an example

$$
\begin{aligned}
\frac{\mathrm{d} q_{x}}{\mathrm{~d} t}= & S_{f x}=\frac{-\tau_{b x}}{\rho}-\frac{\left(\rho_{s}-\rho_{w}\right) g h^{2}}{2 \rho} \frac{\partial c}{\partial x} \\
& -\frac{\left(\rho_{0}-\rho\right)(E-D) q_{x}}{\rho\left(1-p_{0}\right) h},
\end{aligned}
$$

where $q_{x}=h u$. The above equation is then discretized by a full implicit method

$$
\frac{q_{x}^{p}-q_{x}^{k}}{\Delta t}=S_{f x}^{k+1},
$$

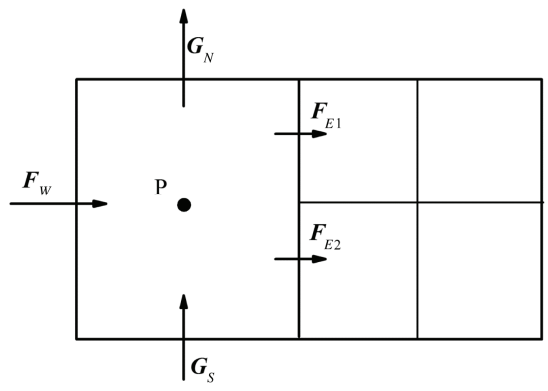

Figure 1 Conservative flux calculation on a non-uniform mesh. 
where the friction source term $S_{f}^{k+1}$ is expressed using a Taylor series as

$$
S_{f x}^{k+1}=S_{f x}^{k}+\left(\partial S_{f x} / \partial q_{x}\right)^{k} \Delta q_{x}+O\left(\Delta q_{x}^{2}\right),
$$

where $\Delta q_{x}=q_{x}^{p}-q_{x}^{k}$. Neglecting the high-order terms and substituting it into eq. (16), the following formula is attained for updating $q_{x}$ to the $p$ time step

$$
q_{x}^{p}=q_{x}^{k}+\Delta t\left(S_{f x} / D_{x}\right)^{k}=q_{x}^{k}+\Delta t F_{x},
$$

where $D_{x}=1-\Delta t\left(\partial S_{f x} / \partial q_{x}\right)^{k}$ is the implicit coefficient and $F_{x}=S_{f x} / D_{x}$ is the friction source term including the implicit coefficient. In order to ensure stability, a limiting value of the implicit friction force must be identified according to the physics of the shallow flows [27]. The maximum effect of the friction force is to constrain the flow, i.e., $q_{x}^{p} q_{x}^{k} \geqslant 0$. Then according to eq. (18), the limiting value of the implicit friction force can be easily derived and is given by

$$
F_{x} \begin{cases}\geqslant-q_{x}^{k} / \Delta t & \text { if } \quad q_{x}^{k} \geqslant 0, \\ \leqslant-q_{x}^{k} / \Delta t \quad \text { if } \quad q_{x}^{k} \leqslant 0 .\end{cases}
$$

If $F_{x}$ computed is beyond the limit, its value is replaced by the critical value $F_{x}=-q_{x}^{k} / \Delta t$ in the actual computation. Similarly, the implicit coefficient in the $y$ direction can be calculated. In eq. (15), the term $\partial c / \partial x$ is discretized using a central difference scheme, as are the diffusion terms in eq. (2d). Notably, there is no clipping treatment to modify flow and sediment variables or fluxes in order to maintain numerical stability. This procedure essentially ensures absolute mass conservation [27].

Bed evolution is computed explicitly from eq. (3),

$$
z_{i c}^{k+1}=z_{i c}^{k}+\Delta t(D-E)_{i c}^{k} /\left(1-p_{0}\right) .
$$

The numerical scheme is explicit. Its stability is controlled by the Courant-Friedrichs-Lewy (CFL) condition, the time step is given by

$$
\Delta t=C r \min \left[\Delta x_{s} /\left(\left|u_{i c}\right|+\sqrt{g h_{i c}}\right), \quad \Delta y_{s} /\left(\left|v_{i c}\right|+\sqrt{g h_{i c}}\right)\right],
$$

where $\mathrm{Cr}$ is the Courant number. It is specified as $\mathrm{Cr}=$ 0.4 for all the test cases below. In fact, the same limit for the $\mathrm{Cr}(\mathrm{Cr} \leqslant 0.5)$ has been imposed before [28] using the MUSCL reconstruction method. In general, the CFL stability condition is valid for non-homogeneous hyperbolic systems with small source terms. Instability of a numerical model built on the non-homogeneous hyperbolic equations can be triggered by large source terms, even for a full implicit numerical model [29]. A common approach to coping with this problem is to use a small time step, corresponding to a small $\mathrm{Cr}$. Also in this regard, if the resistance term for a compound open-channel flow is overestimated by a naïve empirical relationship, it may cause non-physical oscillations, which certainly will spoil the results [30].

\section{Computational tests}

To quantify the difference of two solutions, the average discrepancy $\left(L_{1}\right.$ norm) is defined as

$$
L_{1}=\frac{\sum\left|\chi_{i c}-\bar{\chi}_{i c}\right|}{\sum \hat{\chi}_{i c}},
$$

where $\bar{\chi}$ is the solution of the reference case and $\chi$ is the solution of a separate case for comparison. In the following subsections, the quantity $\chi_{i c}$ chosen for comparison is case specific.

The modelling runs are performed on single core of the Dell Precision T7500 workstation (3.07 GHz CPU with 12 GB RAM) using non-parallelized codes of models based on both fixed and adaptive meshes, except where otherwise specified.

\subsection{Dam break flow over fixed bed}

Bellos et al. [31] carried out a series of dam break flow experiments. The flume was made a gradually converging-diverging plan shape to generate $2 \mathrm{D}$ effects. The flume was $21.2 \mathrm{~m}$ long and a longitudinal slope of $0.6 \%$ was specified. The dam was located at $x=0 \mathrm{~m}$, and instant and full break was accomplished by rapidly lifting the dam (Figure 2). The Manning roughness was calibrated to be 0.012 $\mathrm{s} / \mathrm{m}^{1 / 3}$ [31]. The free outflow boundary condition is applied in the modelling. Fixed meshes (FM) with three different cell sizes and AMR with two different levels (Table 1) are adopted in the modelling. For the adaptive mesh, $\sigma_{1}=0.5$ and $\sigma_{2}=0.2$ are adopted. The coefficient $\gamma$ is presumed to be equal to 0.2 .

Figure 3 shows the computed and measured water depths at four probe locations (Figure 2). The computed water depth agrees well with the measured one at P1, P2 and P3, whilst it is slightly higher than the measured one at P4, which likely results from the uncertain estimation of turbulent diffusion. The relative CPU time for different meshes is shown in Table 1. The unit CPU time is $15.2 \mathrm{~s}$ obtained from FM3. With a finer mesh the relative CPU time is longer for fixed mesh as more computational nodes are involved. The relative CPU time of FM1 is roughly nine times more than that of AMR. Table 2 shows the average discrepancies of different meshes at the four gauges. In this test, $\chi=h$ and the observed depth is defined as reference case. It is seen that the average discrepancy of AMR is very close to that of FM1 (Table 2). This outcome means the 


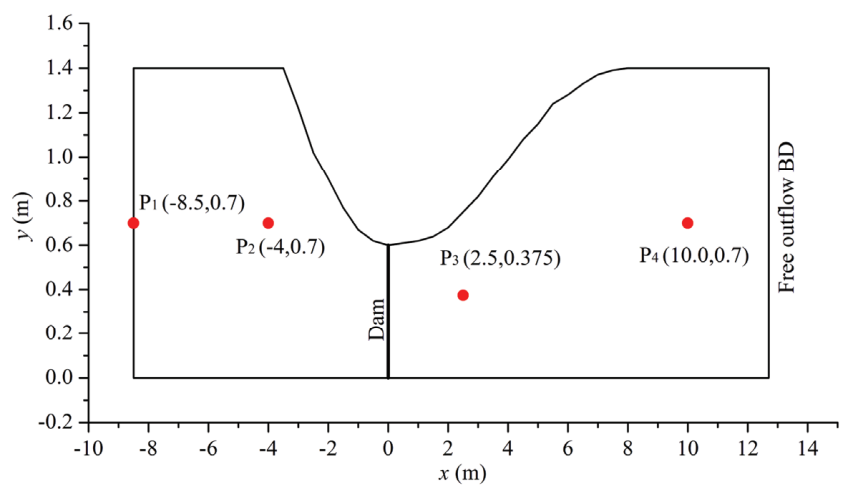

Figure 2 Plane view of the experimental flume and the locations of probes.
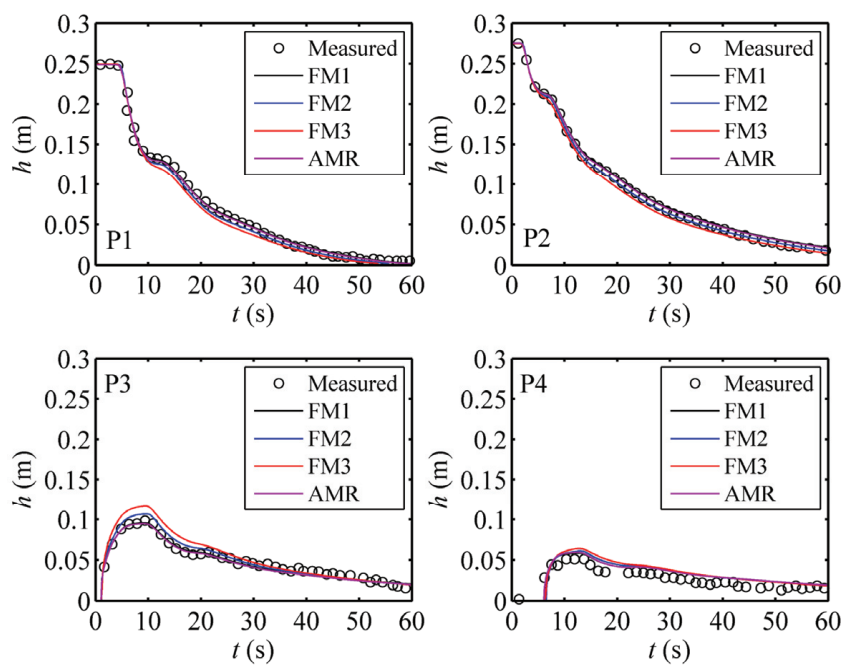

Figure 3 Computed water depths based on the fixed and adaptive meshes along with the observed data.

Table 1 Performance of different meshes for 2D dam break flow over the fixed bed

\begin{tabular}{ccccc}
\hline Cases & Relative CPU time & $\Delta x(\mathrm{~cm})$ & $\Delta y(\mathrm{~cm})$ & Levels \\
\hline FM1 & 54.62 & 2.5 & 2.5 & 1 \\
FM2 & 7.43 & 5 & 5 & 1 \\
FM3 & 1.0 & 10 & 10 & 1 \\
AMR & 5.15 & $2.5,5,10$ & $2.5,5,10$ & 3 \\
\hline
\end{tabular}

Table 2 Average discrepancies of different meshes based on the observed data

\begin{tabular}{ccccc}
\hline Cases & P1 & P2 & P3 & P4 \\
\hline FM1 & 0.1161 & 0.1093 & 0.0931 & 0.2892 \\
FM2 & 0.1438 & 0.1054 & 0.1221 & 0.3248 \\
FM3 & 0.1764 & 0.1495 & 0.1759 & 0.3552 \\
AMR & 0.1130 & 0.1191 & 0.0864 & 0.2879 \\
\hline
\end{tabular}

solution based on AMR achieves the similar resolution to that based on the finest mesh (FM1). The average discrepancy of AMR is smaller than that of FM2, whereas the rela- tive CPU time is less than that of FM2. Moreover, the average discrepancies of fixed mesh increase as the cell size becomes larger, i.e., from FM1 to FM3.

\subsection{Flow due to landslide dam failure}

Cao et al. [32] carried out a series of experiments on flood flows due to landslide failure by overtopping erosion. The experiments were carried out in a large-scale flume of 80 $\mathrm{m} \times 1.2 \mathrm{~m} \times 0.8 \mathrm{~m}$, the bed slope of the flume was 0.001 and the Manning roughness was calibrated to be approximately $0.012 \mathrm{~s} / \mathrm{m}^{1 / 3}$. A weir $0.332 \mathrm{~m}$ high is located at $x=78.0 \mathrm{~m}$, where the outflow is supercritical. A set of 12 automatic water-level probes was used to measure the stage hydrographs at 12 cross-sections along the flume. To demonstrate the performance of the present model, the case without initial breach with uniform sands was revisited, i.e., F-Case 11 in Cao et al. [32]. In this case, the initial upstream and downstream slopes of the dam were $1 / 2$ and $1 / 3$, respectively. The medium diameter of the non-cohesive dam material was $0.8 \mathrm{~mm}$ and the specific gravity of the sediment was 1.65 , invariable in space. The inlet discharge was 0.042 $\mathrm{m}^{3} / \mathrm{s}$, and the initial static water depths immediately upstream and downstream of the dam were $0.054 \mathrm{~m}$ and 0.048 $\mathrm{m}$ respectively. In this test, the modification coefficient $\varphi$ for entrainment is set to be 4.0 following Huang et al. [20], while $\sigma_{1}$ and $\sigma_{2}$ are specified to be 0.4 and 0.01 respectively. The coefficient $\gamma$ vanishes in this case.

Figure 4 shows the computed and measured stage hydrographs at four cross-sections, i.e., CS1 and CS5 that are respectively $22 \mathrm{~m}$ and $1 \mathrm{~m}$ upstream of the dam, and CS8 and CS12 that are respectively 13 and $32.5 \mathrm{~m}$ downstream of the dam. The computed stage hydrographs on both the fixed $(\Delta x=\Delta y=1 \mathrm{~cm})$ and adaptive $(\Delta x=\Delta y=1,2$ and 4 $\mathrm{cm})$ meshes are in agreement with the measured ones, especially at the descending phase. To quantitatively evaluate the discrepancy, $\chi=\eta$ and the measured data are defined as reference case. The average discrepancies of the stage hydrographs at the four cross sections (i.e., CS1, CS5, CS8 and CS12) based on the fixed mesh are $1.67 \%, 1.77 \%$, $0.79 \%$ and $0.88 \%$, while those of the adaptive mesh are $1.34 \%, 1.25 \%, 0.63 \%$ and $0.73 \%$. In this case, the CPU time of the fixed mesh is about $20 \mathrm{~h}$ while that of the adaptive mesh is less than $2 \mathrm{~h}$, leading to a reduction by more than $90 \%$. Figure 5 shows the computed and measured water surface profiles along with the computed bed profile at $t=400$ and $600 \mathrm{~s}$. It is shown that the computed water surface profile agrees well with the measured one. Figure 6 shows the computational meshes at $t=400$ and $600 \mathrm{~s}$. The mesh is finer around the dam and the weir than other parts of the domain and it evolves with time. The mesh at $t=400 \mathrm{~s}$ is dictated by the gradients of water stage and bed elevation, so the finer mesh is shown around the dam and the weir. However, at $t=600 \mathrm{~s}$, the mesh around the dam is dictated 

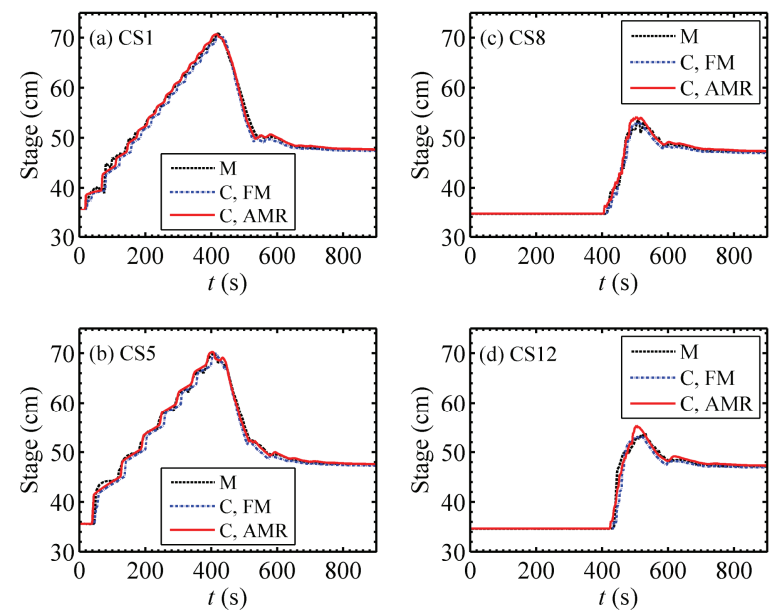

Figure 4 Computed (C) stage hydrographs based on the fixed mesh (FM) and adaptive mesh refinement (AMR) in comparison with the measured (M) data.

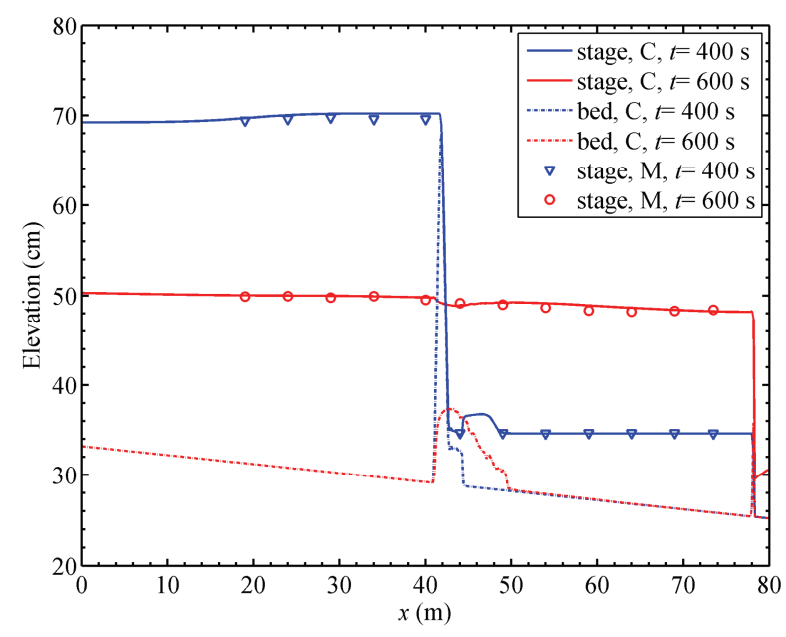

Figure 5 Computed (C) water surface and bed profiles along with the measured (M) water surface profile.
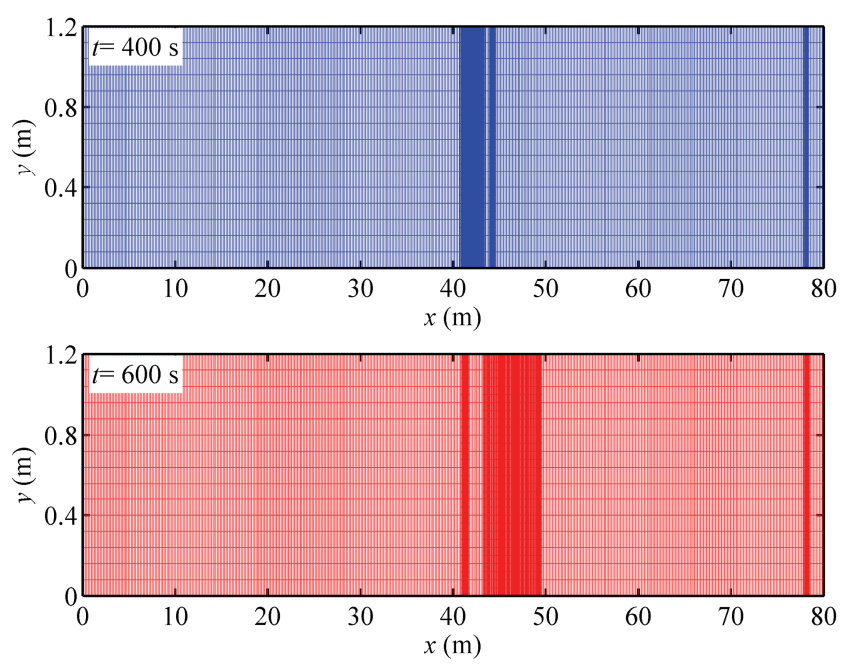

Figure 6 Computational meshes at $t=400$ and $600 \mathrm{~s}$. by the gradient of the bed elevation (Figure 5). This procedure is adopted because the normalized stage gradient is smaller than the threshold value for refinement, whilst the normalized gradient of the bed evolution (or slope) is larger than the threshold value for refinement, and equally importantly, the bed deformation is among the primary processes which we aim to resolve, for which a locally finer mesh is necessary. It suggests that it is justified to incorporate the movable bed elevation in the adaptation indicators.

\subsection{Dam break flow over mobile bed}

A 2D mobile-bed dam-break experiment was performed at UCL-Belgium to investigate the hydrodynamics and morphological evolution [16]. The flume was $3.6 \mathrm{~m}$ wide and about $36 \mathrm{~m}$ long (Figure 7). The partial dam break was represented by rapidly lifting the $1 \mathrm{~m}$ wide gate between the blocks. An $85 \mathrm{~mm}$ thick sand layer was paved extending from $1 \mathrm{~m}$ upstream of the gate to $9 \mathrm{~m}$ downstream of the gate. The fully saturated sediment had a medium diameter $1.61 \mathrm{~mm}$, specific gravity of 2.63, and bed porosity of 0.42 . The experiment involved both initially wet and dry bed scenarios downstream of the gate. Here, only the initially dry case was selected, which was thought to be most difficult case to model [33]. Ultrasonic probes were used to measure the water level at eight gauge points (Figure 7). The Manning roughness $n$ was set as 0.0165 and 0.01 $\mathrm{s} / \mathrm{m}^{1 / 3}$ for the bed covered with and without sand, respectively. The open boundary condition was adopted for the outflow condition. The modification coefficient $\varphi$ for entrainment was set to 1.0 , while $\sigma_{1}$ and $\sigma_{2}$ were specified to 0.2 and 0.01 for the adaptive mesh. The coefficient $\gamma$ is presumed to be equal to 0.2 . Differential meshes were applied, as summarized in Table 3.

The results shown in Figures 8 to 12 pertain to mesh AMR2. Figure 8 shows the computed and measured stage hydrographs at the eight gauges. The computed stage hydrographs at gauges 1 and 4 are about $3.0 \mathrm{~cm}$ lower than the measured ones on average (Figure $8(\mathrm{a})$ ), which is similar to that due to $\mathrm{Wu}$ et al. [33]. One of the possible reasons for the discrepancy is that these two gauges are located at the suddenly expanded corner where the flow is highly three-dimensional and may not be fully represented by shallow water hydrodynamic models. The computed stage hydrographs at other gauges are in reasonable agreement with the measured hydrographs (Figure 8(b)-(d)). To quantitatively evaluate the discrepancy, $\chi=\eta$ and the measured data are defined as a reference case. The average discrepancies of the computed stage hydrographs are $24.19 \%$ at G1(4), 7.66\% at G2(3), 9.30\% at G5(8) and $7.92 \%$ at G6(7) respectively. Figure 9 shows the bed elevation profiles at $y=0.2,0.7$ and $1.45 \mathrm{~m}$. The computed bed elevation profiles agree with the measured values fairly well. The bed deform depth is defined as $\Delta z=\left|z_{\text {final }}-z_{\text {initial }}\right|$, where 


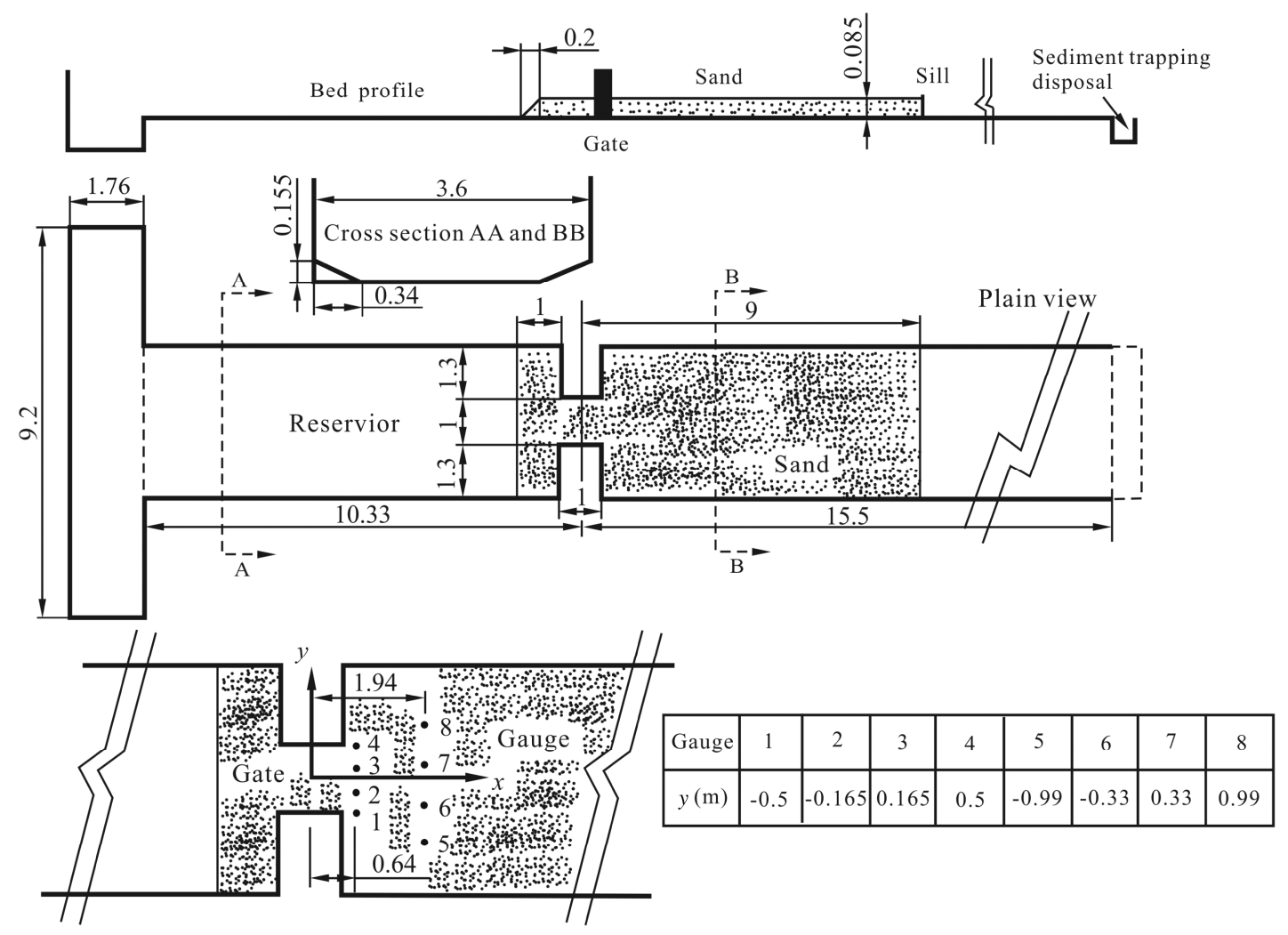

Figure 7 Experiment configuration for 2D dam break over mobile bed.

Table 3 Different meshes for 2D dam-break flow over mobile bed

\begin{tabular}{ccccc}
\hline Cases & Relative CPU time & $\Delta x(\mathrm{~cm})$ & $\Delta y(\mathrm{~cm})$ & Levels \\
\hline FM1 & 56.82 & 1 & 1 & 1 \\
FM2 & 8.04 & 2 & 2 & 1 \\
FM3 & 1.0 & 4 & 4 & 1 \\
AMR1 & 15.86 & 1,2 & 1,2 & 2 \\
AMR2 & 5.41 & $1,2,4$ & $1,2,4$ & 3 \\
\hline
\end{tabular}
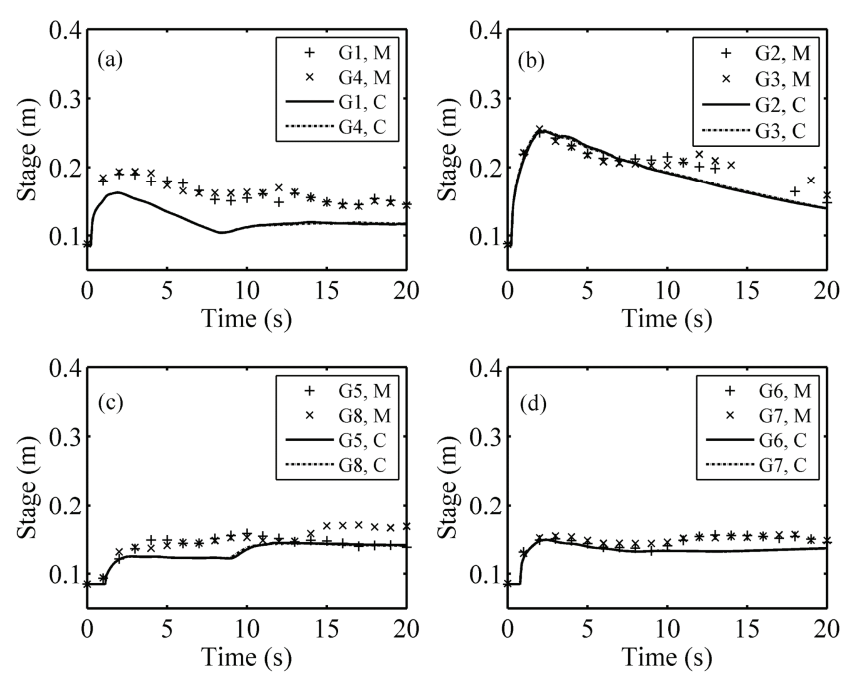

Figure 8 Computed (C) and measured (M) stage hydrographs based on AMR2.
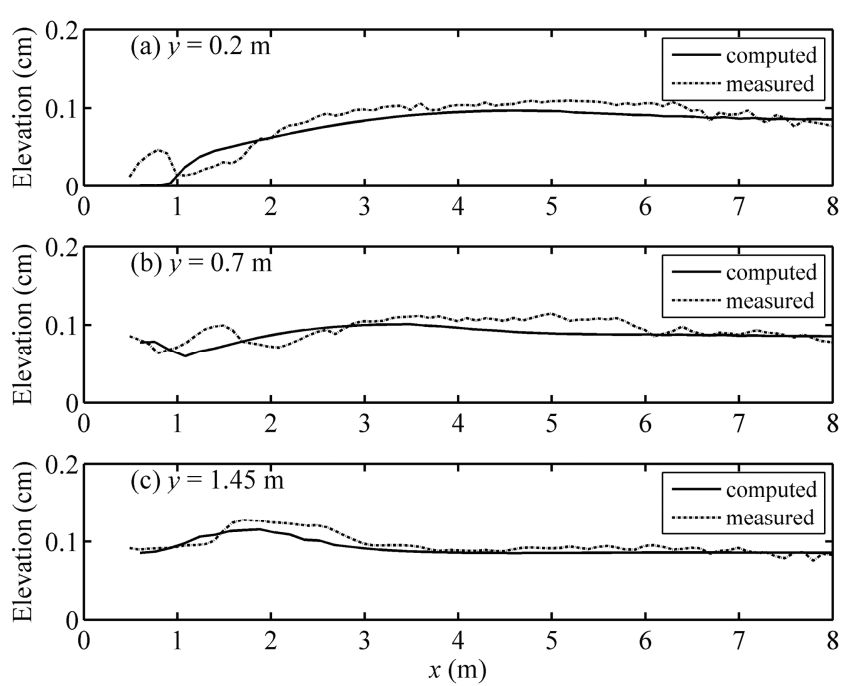

Figure 9 Computed and measured bed elevations at $t=20 \mathrm{~s}$ based on AMR2.

$z_{\text {initial }}$ and $z_{\text {final }}$ are the bed elevation at initial and final instants. To quantify the discrepancy between the computed and measured bed elevation profiles, $\chi=\Delta z$ and that of the measured elevations is defined as reference case. The average discrepancies of the bed elevation at $y=0.2,0.7$ and $1.45 \mathrm{~m}$ are $25.48 \%, 23.95 \%$ and $15.70 \%$ respectively. It should be noted that deviations of the computed water lev- 
els and bed profiles from the measured ones are in a similar magnitude to the discrepancies of water levels and bed profiles among different experiment runs (see Figures 4 and 5 and the related text in ref. [16]).

Figure 10 shows the computed and measured bed topographies at $t=20 \mathrm{~s}$. In the local area near the expanded corner, bed evolution is difficult to accurately model even in a qualitative sense (Figure 10 in ref. [16]), which likely results from the locally strongly three-dimensional flow structure that is essentially not represented by the shallow water hydrodynamic model. Yet, the main morphological evolutions computed and measured are rather similar. Intense bed scouring occurs around the expanded corner from both the computed and measured topographies (note that the initial bed elevation is approximately $0.085 \mathrm{~m}$ ), though the measured bed topography is not as symmetric as the computed one. In this respect, the present model demonstrates promising capacity in reproducing morphological evolution.

Figure 11 shows the computed water surfaces and the meshes at $t=5$ and $20 \mathrm{~s}$. As shown in Figure 11(a) and (c), the gradient of water surface around the expanded corner is very large, which means vertical velocity is nontrivial. Accordingly, the mesh around the expanded corner is locally finer than elsewhere (Figures 11(b), (d)).

Figure 12 shows the variation of $\sigma$ based on stage, bed elevation and volumetric sediment concentration at three points $\mathrm{P} 1(0.62,-0.92), \mathrm{P} 2(3.1,-1.28)$ and $\mathrm{P} 3(-0.8,1.76)$ respectively. As shown in Figure 12(a), the $\sigma$ based on stage is larger than $\sigma_{1}=0.2$ between 6 and $20 \mathrm{~s}$, while those based on concentration and bed elevation are lower than 0.2 . Accordingly, the cell where P1 is located is subject to refinement between 6 and $20 \mathrm{~s}$ as dictated by $\sigma$ based on stage. Indeed, cell refinement is necessary as determined by $\sigma$ based on concentration at P2 (between 5.3 and $7.5 \mathrm{~s}$ approximately), (see Figure 12(b)). At P3, refinement is necessary due to $\sigma$ based on bed elevation, which is clear from Figure 12(c). Therefore, it is important to introduce concentration as a variable to dictate the mesh adaptation.

Figure 13 shows the computed stage hydrographs on different meshes (Table 3) and those measured at the eight gauges (Figure 7). Due to the symmetric locations of the gauges, the computed stage hydrographs at only one of the symmetric gauges are shown. Appreciable discrepancy can be spotted among the computed stage hydrographs based on different meshes, which suggests that the mesh can modify the stage hydrographs to a certain extent. Yet, all the computed stage hydrographs from different meshes deviate from the measured ones to a similar extent. Unfortunately, it remains hard to unravel what factors lead to the deviation, given the current knowledge on the interactions of dam break floods with an erodible bed. The need for further fundamental research into the phenomenon is warranted. Nevertheless, the largely enhanced computational efficiency is evident based on AMR when compared to the fixed meshes (Table 3).
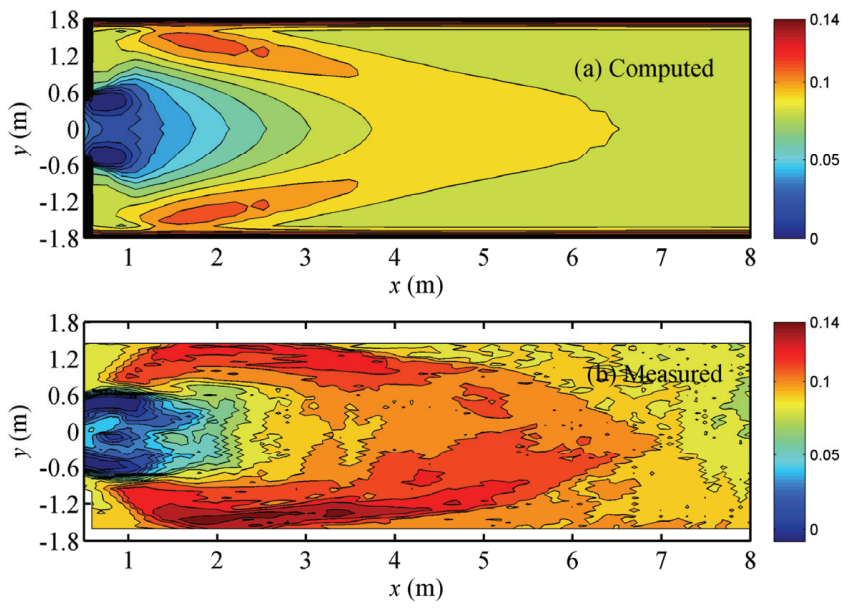

Figure 10 Computed (a) and measured (b) bed topographies at $t=20 \mathrm{~s}$ based on AMR2.
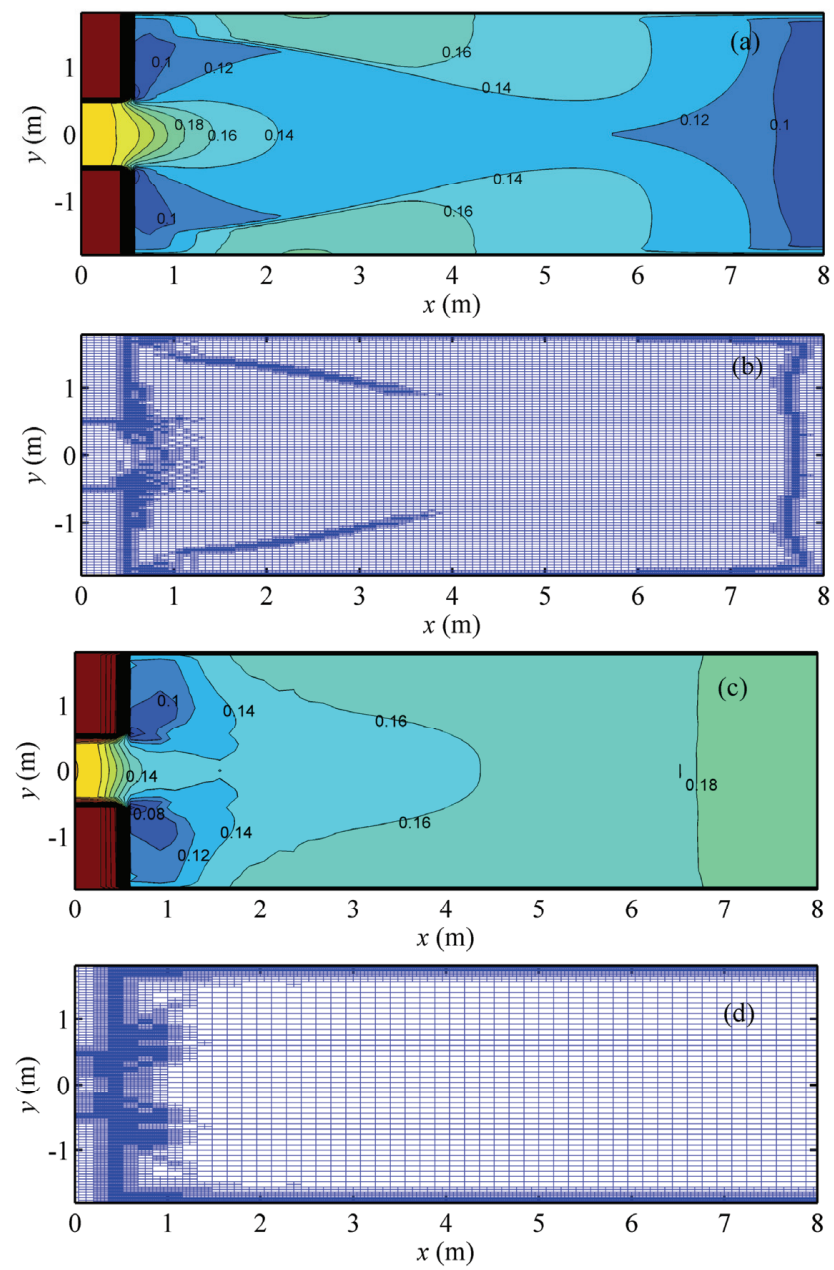

Figure 11 Computed water surface (a), (c) and adaptive mesh (b), (d) at $t=5$ and $20 \mathrm{~s}$ based on AMR2.

\subsection{Megaflood due to glacier dam-break over mobile bed}

The glacier dam-break flood in the Altai Mountains was one 

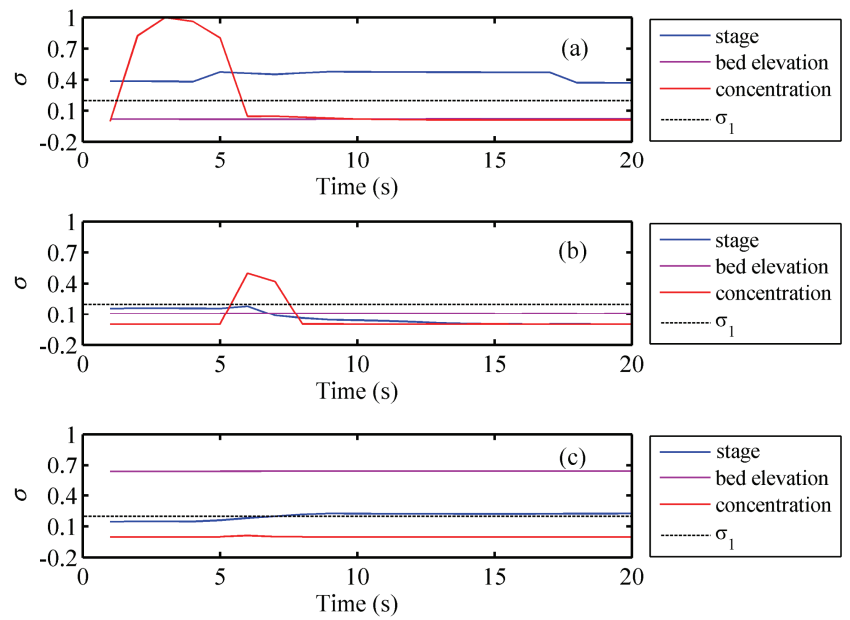

Figure $12 \sigma$ variation for stage, bed elevation and concentration at P1 (a); P2 (b) and P3 (c).
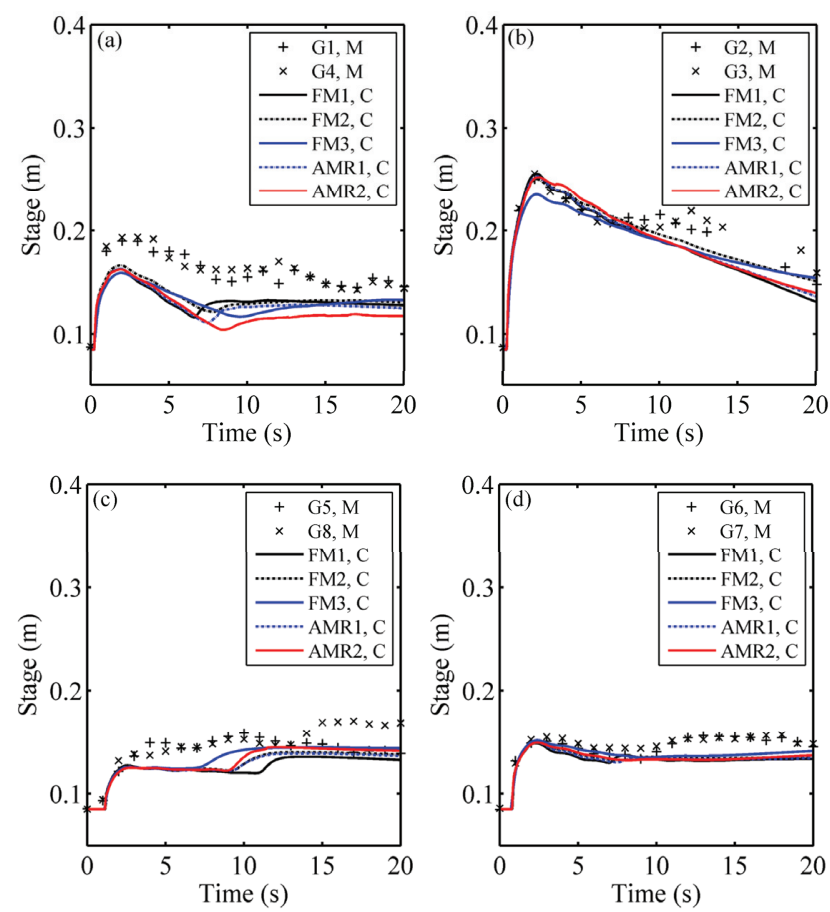

Figure 13 Computed (C) and measured (M) stage hydrographs on different meshes.

of the largest floods on the Earth [34]. The background of this megaflood was presented before [34-36]. This megaflood was modelled by Carling et al. [36] using integrated 1D and 2D hydraulic models over a presumed fixed bed. Yet, a megagflood is certainly highly energetic, capable of triggering very active sediment transport and intense geomorphic change [35], which in turn modifies the flood flow significantly. Even a single flood event can result in rapid formation of a modern bedrock canyon [37]. Therefore, the modelling of sediment transport and bed evolution induced by the flood is important. The Manning roughness $n$ is set to
$0.05 \mathrm{~s} / \mathrm{m}^{1 / 3}$ following Carling et al. [36] and the medium sediment diameter is $120 \mathrm{~mm}$. The maximum depth of the bed, which can be eroded, is confined to $50 \mathrm{~m}$ according to the field survey. The modification coefficient $\varphi$ for entrainment is set to 1.0 and the coefficient $\gamma$ is specified to 0.2 . The "minimum model" proposed by Walder and Costa [38] is adopted to specify the discharge hydrograph (with peak discharge being about $9.45 \times 10^{6} \mathrm{~m}^{3} / \mathrm{s}$ ) at the upstream boundary (ice dam site, $x=220 \mathrm{~km}$ in Figure 14). Transmissive boundary conditions (i.e., gradients of velocity and depth are set to zero) are applied at the downstream boundary if the cross-sectional Froude number is greater than unity $[39,40]$; otherwise, a local uniform flow condition is imposed. The models based on the both fixed and adaptive meshes are adopted. For the modelling based on the adaptive mesh, two levels (i.e., level 0 and 1) are used, and the DEM resolution is $50 \mathrm{~m} \times 50 \mathrm{~m}$, with $\sigma_{1}$ and $\sigma_{2}$ specified to be 0.2 and 0.01 . The minimum cell size of the adaptive mesh is $50 \mathrm{~m} \times 50 \mathrm{~m}$, which equals to the cell size of fixed mesh. The megaflood flowed in a large-scale domain, which involves about 3.75 million and more than 1.5 million computational cells for the fixed and adaptive meshes, respectively. In order to enhance the computational efficiency, the codes on both the fixed and adaptive meshes are parallelized using Open specifications for Multi-Processing (OpenMP) method.

To quantitatively evaluate the discrepancies between the results on different meshes, the water depth corresponding to the maximum stage is chosen as $\chi$ in eq. (22) and that of the fixed mesh is defined as the reference case. The average discrepancy based on the adaptive mesh is $7.031 \%$. It is shown in Figure 14 that the final bed topographies based on the fixed and adaptive meshes are in fairly good agreement with each other. Quantitatively, $\chi=\Delta z$ and that of the fixed mesh is defined as the reference case. The average discrepancy of the bed deformation depth based on the adaptive mesh is $0.2 \%$. Models based on both the fixed and adaptive meshes are performed on 12 cores CPU of the workstation. It costs more than $30 \mathrm{~d}(720 \mathrm{~h})$ for a run from the model based on the fixed mesh, while only about $5 \mathrm{~d}(120 \mathrm{~h})$ are required for the model on the adaptive mesh. The results demonstrate that the model based on the adaptive mesh can save the computational time significantly while achieving similar accuracy to the fixed mesh.

Figure 15 shows the comparison of the maximum water stage profiles from both the fixed and adaptive meshes. Unfortunately, the observed data of the maximum stage for the historic megaflood are unavailable due to obvious reasons. Therefore the elevations of the deposits and bars, emanating from post-flood geological surveys, are included for comparison. The main routing of the flood started from the ice dam site (distance downstream of ice dam is $0 \mathrm{~km}$ in Figure 15) along the Chuja River, extended to the Katun River and eventually flowed downstream to the lower Katun 

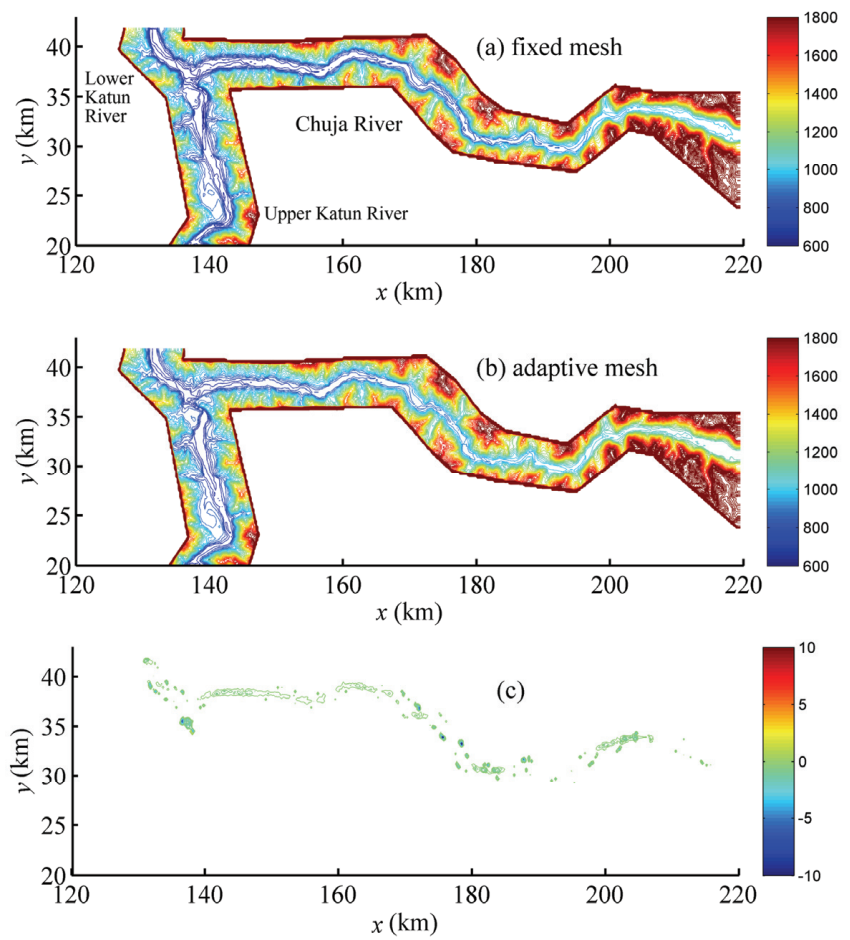

Figure 14 Final topography modelled based on (a) the fixed, and (b) the adaptive meshes and (c) the discrepancy.

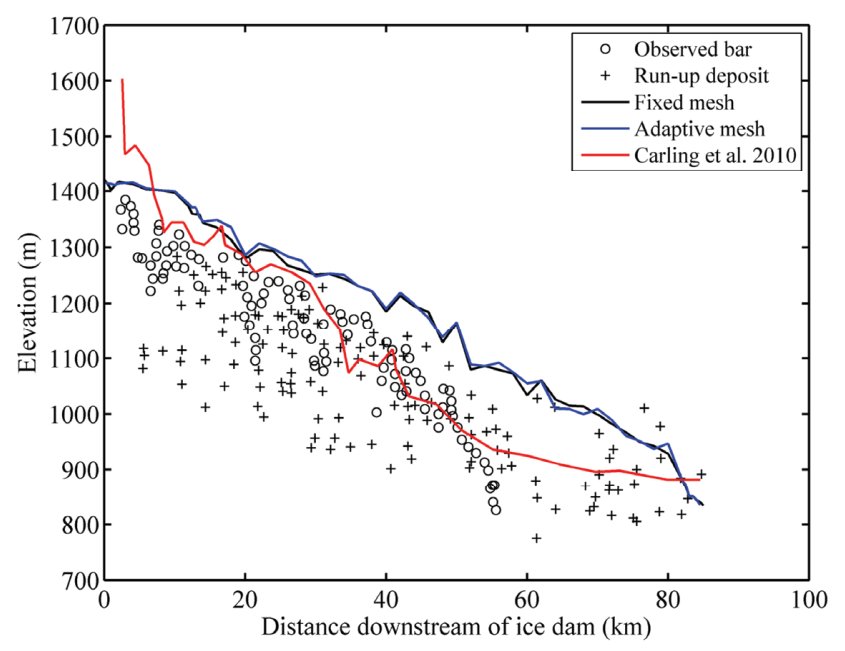

Figure 15 Computed maximum stage profiles and field data.

River. It is shown that the computed maximum stage profile based on the adaptive mesh is in line with that based on the fixed mesh and both agree with the field data reasonably well. It should be noted that the maximum stage is lower than the run-up deposits around $80-85 \mathrm{~km}$ in Figure 15. This may be attributed to the uncertainty that is inevitable because no data are available for specifying the downstream boundary condition.

It is also interesting to compare the results of the present work to those of Carling et al. [36]. The maximum stage profile in between 10 and $20 \mathrm{~km}$ downstream the ice dam, computed by the present model (Figure 15), is close to that of Carling et al. [36]. In between 30 and $85 \mathrm{~km}$, the computed maximum stage from Carling et al. [36] is significantly lower than the observed bars and run-up deposits, which is physically unrealistic. In contrast, the maximum stage profile in the same range resolved by the present model (Figure 15) is qualitatively consistent with the observed data. Several factors are responsible for the differences between the results from Carling et al. [36] and the present work. First of all, in Carling et al. [36] only the flood water flow is modelled without taking into account the active sediment transport and substantial morphological changes. However, the strongly interactive processes of the flood flow, sediment transport and morphological evolution are modelled in a coupled manner in the present model. Also, the numerical algorithms in the two models are different, which also contributes to the differences between the two models. Additionally, the boundary conditions (especially the downstream boundary condition) may be different in the two models as no direct information is available.

Figure 16 shows the flow velocity fields around the confluence of the Chuja River and Katun River at two instants, computed by the present model. It is shown that at $t=1.2 \mathrm{~h}$ (Figure 16(a)), the flood propagated into both the lower and upper reaches of Katun River and the velocities in the Chuja River and the lower Katun River were considerably higher than those in the upper Katun River. At $t=24 \mathrm{~h}$, the velocities in the upper Katun River were about two orders of magnitude lower than those in the lower Katun River and Chuja River. Thus Figure 15(c) is presented to better show the velocity field in the upper Katun River, which is essentially zoomed in from Figure 16(b). The main routing of the flood remained to be the Chuja River and the lower Katun River (Figure 16(b)) and the impounded water in the upper Katun River flowed back downstream to the lower Katun River at $t=24 \mathrm{~h}$. The velocity fields at the two instants clearly demonstrate that the bifurcated flows and backwater effects are resolved by the present model.

\section{Conclusion}

A coupled 2D shallow water hydrodynamic and noncapacity sediment transport model has been developed based on the adaptive structured non-uniform mesh. Stage gradient and sediment concentration gradient, which are important in cases involving rapid bed evolution and intense sediment transport, are incorporated in the adaptation indexes. A series of computational tests show that the proposed model has advantage in saving CPU time $80 \%$ to 93\% as contrasted against its counterparts based on the fixed meshes, while similar accuracy is maintained. It should find wide applications in modelling large-scale shallow water flows featuring sediment transport and morpho- 

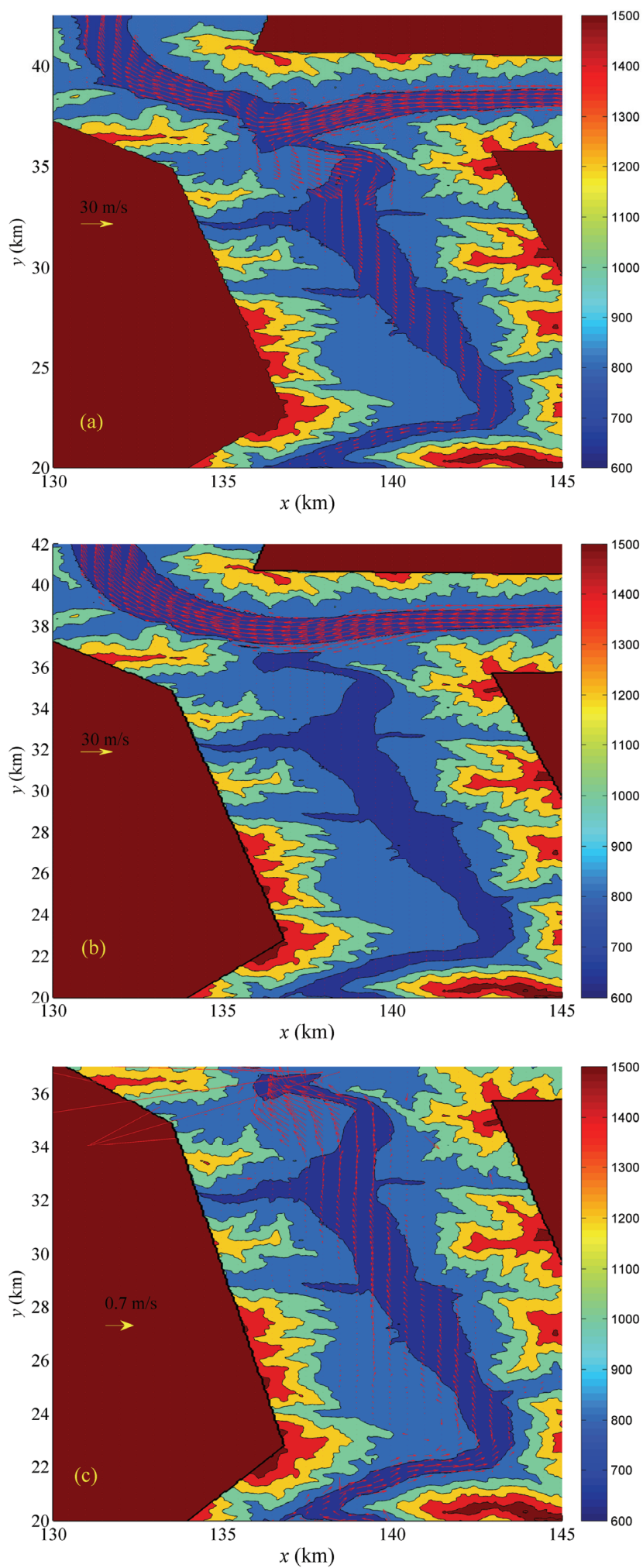

Figure 16 Velocity distribution around the confluence of the Chuja River and Katun River (a) $t=1.2 \mathrm{~h}$ and (b) $t=24 \mathrm{~h}$, as well as in upper Katun River (c) at $t=24 \mathrm{~h}$.

logical changes. To minimize model uncertainty, enhanced understanding of sediment transport mechanics is essential, which necessitates fundamental studies on the interactions between turbulent flows and grains $[41,42]$.

This work was supported by the National Natural Science Foundation of China (Grant Nos. 11172217, 51279144 \& 11432015).

1 Duan J G, Nanda S K. Two-dimensional depth-averaged model simulation of suspended sediment concentration distribution in a groyne field. J Hydrol, 2006, 327: 426-437

2 Zhang S, Duan J G. 1D finite volume model of unsteady flow over mobile bed. J Hydrol, 2011, 405: 57-68

3 Denlinger R P, O'Connell D R H. Simulations of cataclysmic outburst floods from Pleistocene Glacial Lake Missoula. Geol Soc Am Bull, 2010, 122: 678-689

4 Liang Q. A structured but non-uniform Cartesian grid-based model for the shallow water equations. Int J Numer Meth Fl, 2011, 66: 537-554

5 Caviedes-Voullième D, García-Navarro P, Murillo J. Influence of mesh structure on 2D full shallow water equations and SCS Curve Number simulation of rainfall/runoff events. J Hydrol, 2012, 448449: 39-59

6 Cook A, Merwade V. Effect of topographic data, geometric configuration and modeling approach on flood inundation mapping. J Hydrol, 2009, 377: 131-142

7 Gallegos H A, Schubert J E, Sanders B F. Two-dimensional, high-resolution modeling of urban dam-break flooding: A case study of Baldwin Hills, California. Adv Water Resour, 2009, 32: 1323-1335

8 Crowder D, Diplas P. Using two-dimensional hydrodynamic models at scales of ecological importance. J Hydrol, 2000, 230: 172-191

9 Berger M J, Oliger J. Adaptive mesh refinement for hyperbolic partial differential equations. J Comput Phys, 1984, 53: 484-512

10 George D L. Adaptive finite volume methods with well-balanced Riemann solvers for modeling floods in rugged terrain: Application to the Malpasset dam-break flood (France, 1959). Int J Numer Meth Fl, 2010, 66: 1000-1018

11 Greaves D M, Borthwick A G L. Hierarchical tree-based finite element mesh generation. Int J Numer Meth Eng, 1999, 45: 447-471

12 Chen A S, Evans B, Djordjević S, et al. Multi-layered coarse grid modelling in 2D urban flood simulations. J Hydrol, 2012, 470-471: $1-11$

13 Yiu K F C, Greaves D M, Cruz S, et al. Quadtree grid generation: Information handling, boundary fitting and CFD applications. Comput Fluids, 1996, 25: 759-769

14 Benkhaldoun F, Sari S, Seaid M. A flux-limiter method for dam-break flows over erodible sediment beds. Appl Math Model, 2012, 36: 4847-4861

15 Cao Z, Pender G, Wallis S, et al. Computational dam-break hydraulics over erodible sediment bed. J Hydraul Eng ASCE, 2004, 130: 689-703

16 Soares-Frazão S, Canelas R, Cao Z, et al. Dam-break flows over mobile beds: experiments and benchmark tests for numerical models. $\mathrm{J}$ Hydraul Res, 2012, 50: 364-375

17 Wang J P, Liang Q. Testing a new adaptive grid-based shallow flow model for different types of flood simulations. J Flood Risk Manag, 2011, 4: 96-103

18 Benkhaldoun F, Sahmim S, Seaïd M. A two-dimensional finite volume morphodynamic model on unstructured triangular grids. Int $\mathrm{J}$ Numer Meth Fl, 2010, 63: 1296-1327

19 Zhang M, Wu W M. A two dimensional hydrodynamic and sediment transport model for dam break based on finite volume method with quadtree grid. Appl Ocean Res, 2011, 33: 297-308

20 Huang W, Cao Z, Yue Z, et al. Coupled modelling of flood due to natural landslide dam breach. Proc Inst Civil Eng-Water Manag, 2012, 165: 525-542 
21 Zhang R, Xie J. Sedimentation research in China: Systematic selections. Beijing: China Water and Power Press, 1993

22 Cao Z, Yue Z, Pender G. Landslide dam failure and flood hydraulics. Part II: coupled mathematical modelling. Nat Hazards, 2011, 59: 1021-1045

23 Meyer-Peter E, Müller R. Formulas for Bed-Load Transport, in: IAHR 2nd meeting, IAHR Stockholm, 1948, 39-64

24 Benkhaldoun F, Elmahi I, Seaïd M. Well-balanced finite volume schemes for pollutant transport by shallow water equations on unstructured meshes. J Comput Phys, 2007, 226: 180-203

25 Hirsch C. Numerical Computation of Internal and External Flows (Vol. 1): Fundamentals of Numerical Discretization. London: John Wiley \& Sons, 1990

26 Toro E. Shock-capturing methods for free-surface shallow flows. London: John Wiley, 2001

27 Liang Q, Marche F. Numerical resolution of well-balanced shallow water equations with complex source terms. Adv Water Resour, 2009, 32: 873-884

28 Berthon C. A stable gradient reconstruction for the MUSCL schemes applied to systems of conservation laws. Flow Turbul Combust, 2006, 76: 343-351

29 Venutelli M. Stability and accuracy of weighted four-point implicit finite difference schemes for open channel flow. J Hydraul Eng ASCE, 2002, 128: 281-288

30 Cao Z, Meng J, Pender G, et al. Flow resistance and momentum flux in compound open channels. J Hydraul Eng ASCE, 2006, 132: $1272-1282$

31 Bellos C V, Soulis V, Sakkas J G. Experimental investigation of two-dimensional dam-break induced flows. J Hydraul Res, 1992, 30: 47-63

32 Cao Z, Yue Z, Pender G. Landslide dam failure and flood hydraulics.
Part I: Experimental investigation. Nat Hazards, 2011b, 59: 1003-1019

33 Wu W, Marsooli R, He Z. Depth-averaged two-dimensional model of unsteady flow and sediment transport due to noncohesive embankment break/breaching. J Hydraul Eng, 2012, 138: 503-516

34 Herget J. Reconstruction of Pleistocene ice-dammed lake outburst floods in the Altai Mountains, Siberia. Geol Soc Am Special Papers, 2005, 386: 1-2

35 Carling P. Morphology, sedimentology and palaeohydraulic significance of large gravel dunes, Altai Mountains, Siberia. Sedimentology, 1996, 43: 647-664

36 Carling P, Villanueva I, Herget J, et al. Unsteady 1D and 2D hydraulic models with ice dam break for Quaternary megaflood, Altai Mountains, southern Siberia. Global Planet Change, 2010, 70: 24-34

37 Walder J, Costa J. Outburst floods from glacier-dammed lakes: The effect of mode of lake drainage on flood magnitude. Earth Surf Proc Land, 1996, 21: 701-723

38 Lamb M P, Fonstad M A. Rapid formation of a modern bedrock canyon by a single flood event. Nat Geosci, 2010, 3: 477-481

39 Liao C B, Wu M S, Liang S J. Numerical simulation of a dam break for an actual river terrain environment. Hydrol Process, 2007, 21: $447-460$

40 Zhou J G, Causon D M, Mingham C G, et al. Numerical prediction of dam-break flows in general geometries with complex bed topography. J Hydraul Eng ASCE, 2004, 130: 332-340

41 Ji C N, Ante M, Eldad A, et al. Numerical investigation of particle saltation in the bed-load regime. Sci China Tech Sci, 2014, 58: $1500-1511$

42 Zhang L, Zhong D Y, Wu B S. Particle inertia effect on sediment dispersion in turbulent open-channel flows. Sci China Tech Sci, 2014, 58: 1977-1987 